\title{
Quarkonium correlators at finite temperature and potential models
}

\author{
Ágnes Mócsy \\ RIKEN-BNL Research Center, Brookhaven National Laboratory, Upoton, NY, USA \\ E-mail: mocsy@quark.phys.bnl.gov
}

\section{Péter Petreczky*,}

Department of Physics, Brookhaven National Laboratory, Upton, NY, USA

E-mail: petreczk@bnl.gov

\begin{abstract}
We discuss the calculations of quarkonium spectral functions in potential models and their implications for the interpretation of the lattice data on quarkonium correlators. In particular, we find that melting of different quarkonium states does not lead to significant change in the Euclidean time correlators. The large change of the quarkonium correlators above deconfinement observed in the scalar and axial-vector channels appears to be due to the zero mode contribution.
\end{abstract}

The XXV International Symposium on Lattice Field Theory

July 30-4 August 2007

Regensburg, Germany

\footnotetext{
${ }^{*}$ Speaker.
} 


\section{Introduction}

It was argued long ago that melting of quarkonia above the deconfinement transition can serve as a signature of quark-gluon plasma formation in heavy ion collisions [1]. The basic idea behind this proposal was that due to color screening the potential between a quark and anti-quark will not provide sufficient binding at high temperature. This problem can be formulated more rigorously in terms of quarkonium spectral functions, which can be, in principle, extracted from Euclidean-time meson correlation functions calculated on the lattice. Attempts doing this based on the Maximum Entropy Method (MEM) have been discussed over the last few years. The initial interpretation of data led to the conclusion that the $1 S$ charmonium states survive in the deconfined medium up to temperatures of about $1.6 T_{c}$, with $T_{c}$ being the transition temperature $[2,3,4,5]$. Recent analysis, however, has shown that, although MEM can be used to extract reliably quarkonium spectral functions at zero temperature, at finite temperature it has severe limitations [6].

At zero temperature quarkonium spectrum is well described in non-relativistic potential models. Since the seminal paper by Matsui and Satz the problem of charmonium dissolution has been studied in potential models $[7,8,9,10,11,12,13,14,15,16]$. While the early studies used phenomenological potential, more recent studies rely on static quark anti-quark free energy calculated on the lattice. In fact significant progress has been made in understanding the in-medium modification of inter-quark forces via lattice calculations of the free energy of static quark anti-quark pair. Calculations have been done in pure gluodynamics, 3-flavor and 2-flavor QCD [17, 18, 19], and preliminary results are also available in the physically relevant case of one heavy strange quark and two light quarks [20, 21] (the light quark masses correspond to pion mass of about $220 \mathrm{MeV}$ ).

Recently attempts to calculate quarkonium properties at finite temperature using resummed perturbation theory have been made $[22,23]$. Resummed perturbation theory appears to be successful in calculations of bulk thermodynamics properties [24, 25, 26].

Since the lattice calculations of spectral functions have severe limitations, in [12, 13] it has been pointed out, that comparison between the lattice data and potential models should be done in terms of the Euclidean time correlators, for which the numerical results are much more reliable. Recent studies following this line have also been presented in Refs. [14, 15, 16]. In this contribution we discuss the calculation of quarkonium spectral functions in a potential model, which uses the lattice data of the free energy of a static quark anti-quark pair. Since reliable calculations of the quarkonium correlators are available only in quenched approximation we consider QCD with only heavy quarks. Further details about this approach can be found in Ref. [27].

\section{Charmonium Spectral Functions in Potential Model}

For heavy quarks the spectral function can be related to the non-relativistic Green's function

$$
\begin{gathered}
\sigma(\omega)=\left.K \frac{6}{\pi} \operatorname{Im} G^{n r}\left(\vec{r}, \vec{r}^{\prime}, E\right)\right|_{\vec{r}=\vec{r}^{\prime}=0}, \\
\sigma(\omega)=\left.K \frac{6}{\pi} \frac{1}{m^{2}} \operatorname{Im} \vec{\nabla} \cdot \vec{\nabla}^{\prime} G^{n r}\left(\vec{r}, \vec{r}^{\prime}, E\right)\right|_{\vec{r}=\vec{r}^{\prime}=0},
\end{gathered}
$$

for $S$-wave, and $P$-wave charmonia, respectively. Here $E=\omega-2 m$. At leading order $K=1$. Relativistic and higher order perturbative corrections will lead to a value different from unity [27]. 
The non-relativistic Green's function satisfies the Schrödinger equation

$$
\left[-\frac{1}{m} \vec{\nabla}^{2}+V(r)-E\right] G^{n r}\left(\vec{r}, \vec{r}^{\prime}, E\right)=\delta^{3}\left(r-r^{\prime}\right) .
$$

The numerical method for solving this equation is presented in [27]. At zero temperature we use the Cornell potential $V(r)=-\alpha / r+\sigma r$ with parameters motivated by lattice results on static potential : $\alpha=\pi / 12$ and $\sigma=(1.65-\pi / 12) r_{0}^{-2}$. In the actual calculations we use a potential which is screened beyond some distance $r>r_{\text {med }}$ with screening length $\mu$ to mimic many body effects at large energies (see Ref. [27] for further details). At finite temperature we use a potential motivated by lattice results on the singlet free energy of a static quark anti-quark pair and which is defined in section IV of Ref. [27]. At large energies, away from the threshold, the non-relativistic treatment is not applicable. The spectral function in this domain, however, can be calculated using perturbation theory. We smoothly match the non-relativistic calculation of the spectral function to the relativistic perturbative result [27]. Euclidean time correlators $G(\tau, T)$ at some temperature $T$ can be calculated from the spectral functions using the integral representation

$$
G(\tau, T)=\int_{0}^{\infty} d \omega \sigma(\omega, T) K(\omega, \tau, T) .
$$

Here the integration kernel is

$$
K(\omega, \tau, T)=\frac{\cosh \omega(\tau-1 /(2 T))}{\sinh (\omega /(2 T))}
$$

\section{Correlators at Zero Temperature}

In this section we discuss the comparison of the model calculations with zero temperature lattice data from isotropic lattices [4]. The lattice spacing has been fixed using the Sommer-scale $r_{0}=0.5 \mathrm{fm}$. Its value is slightly larger than the one used in Ref. [4], since there the string tension of $\sqrt{\sigma}=420 \mathrm{MeV}$ has been used to set the scale. Calculations have been done at the charm quark mass which corresponds to an $\eta_{c}$ mass of about $4 \mathrm{GeV}$. The renormalization constants of the lattice operators have been calculated in 1-loop tadpole improved perturbation theory (see Ref. [4] for further details). The $K$ factors in Eq. (2.2) have been chosen such that at large distances the correlators calculated in potential models agree with the lattice results. In Fig. 1 we show the pseudo-scalar correlator calculated on the lattice and in the potential model for several screening parameters, together with the corresponding spectral functions. As one can see from the figure the choice of the ad-hoc screening parameters have almost no effect on the Euclidean correlator. We see a reasonably good agreement between the lattice data and potential model calculations. Also shown in the figure is the correlator corresponding only to the non-relativistic spectral function. At small Euclidean times this falls below the lattice data by more than an order of magnitude. Thus correlators calculated on the lattice are sensitive to the relativistic continuum part of the spectral functions. Similar analysis of the correlators have been done in the vector, axial vector and scalar channels. 


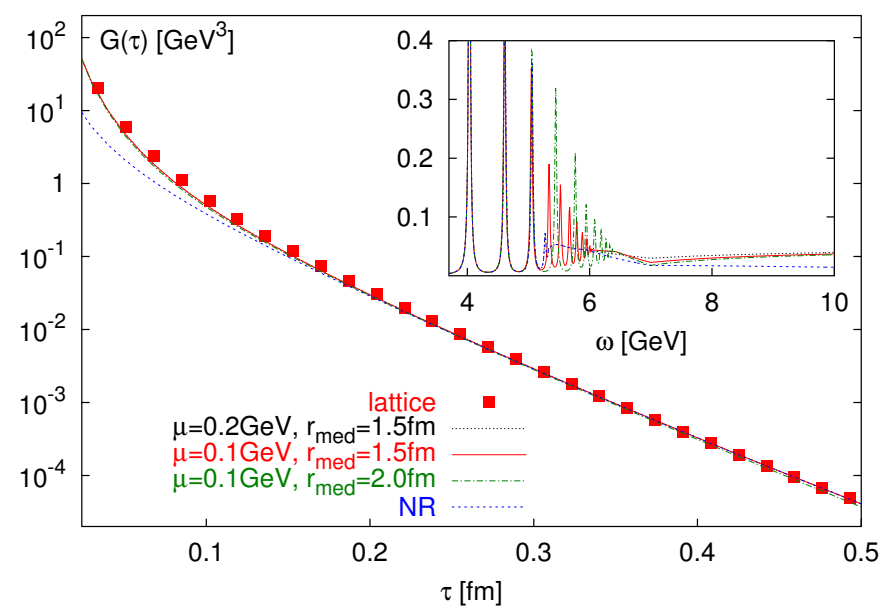

Figure 1: The pseudo-scalar charmonium correlator calculated in our model and compared to the lattice data of Ref. [4]. In the inset, the corresponding spectral functions $\sigma(\omega) / \omega^{2}$ are shown.

\begin{tabular}{|ccccc|}
\hline$T$ & 0 & $1.2 T_{c}$ & $1.5 T_{c}$ & $2.0 T_{c}$ \\
\hline$s_{0}$ & 10.975 & 9.541 & 9.462 & 9.384 \\
$M(1 S)$ & 9.405 & 9.390 & 9.374 & 9.343 \\
$E_{\text {bind }}$ & 1.570 & 0.151 & 0.088 & 0.041 \\
\hline
\end{tabular}

Table 1: The mass and the binding energy of the $1 S$ bottomonium state at different temperatures, and the continuum threshold.

\section{Temperature-dependence of Quarkonium Correlators}

In this section we study the temperature-dependence of quarkonium spectral functions and correlators. Since the correlators depend on the temperature through the integration kernel and the spectral functions, it is customary to study the temperature dependence of the correlators in terms of the ratio $G(\tau, T) / G_{\text {rec }}(\tau, T)$, where

$$
G_{r e c}(\tau, T)=\int_{0}^{\infty} d \omega \sigma(\omega, T=0) K(\omega, \tau, T) .
$$

This way the trivial temperature dependence due to the integration kernel is taken care of. Also many uncertainties of the lattice calculations cancel out in this ratio. The finite temperature spectral functions are shown in Fig. 2 for the pseudo-scalar channel. The $1 \mathrm{~S}$ charmonium state is melted at $1.2 T_{c}$. We see, however, a large enhancement of the spectral functions near the threshold. Note, that the height of the spectral functions near the threshold is comparable to the height of the bump in the spectral function calculated from MEM [6]. It is therefore possible that the bump of the spectral functions calculated from lattice correlators using MEM actually corresponds to a threshold enhancement, and was mistakenly interpreted as the $1 S$ state. In the case of bottomonium all states, except the $1 S$ state, are dissolved above the deconfinement transition. The $1 S$ state can survive as a resonance until temperatures of about $2 T_{c}$. Note, however, that the binding energy of the $1 S$ bottomonium is significantly reduced due to color screening, as shown in Table 1 . The binding energy is defined as the distance between the continuum threshold $s_{0}$ and the bound state peak. 
Due to the small binding energy the $1 \mathrm{~S}$ state will acquire a sizable thermal width, and may not show up as the resonance in the corresponding spectral function. Therefore, the actual dissolution temperature of the $1 S$ bottomonium will be smaller then the one estimated based on the simple potential model calculations which do not include the effect of the thermal width [28]. In the insets
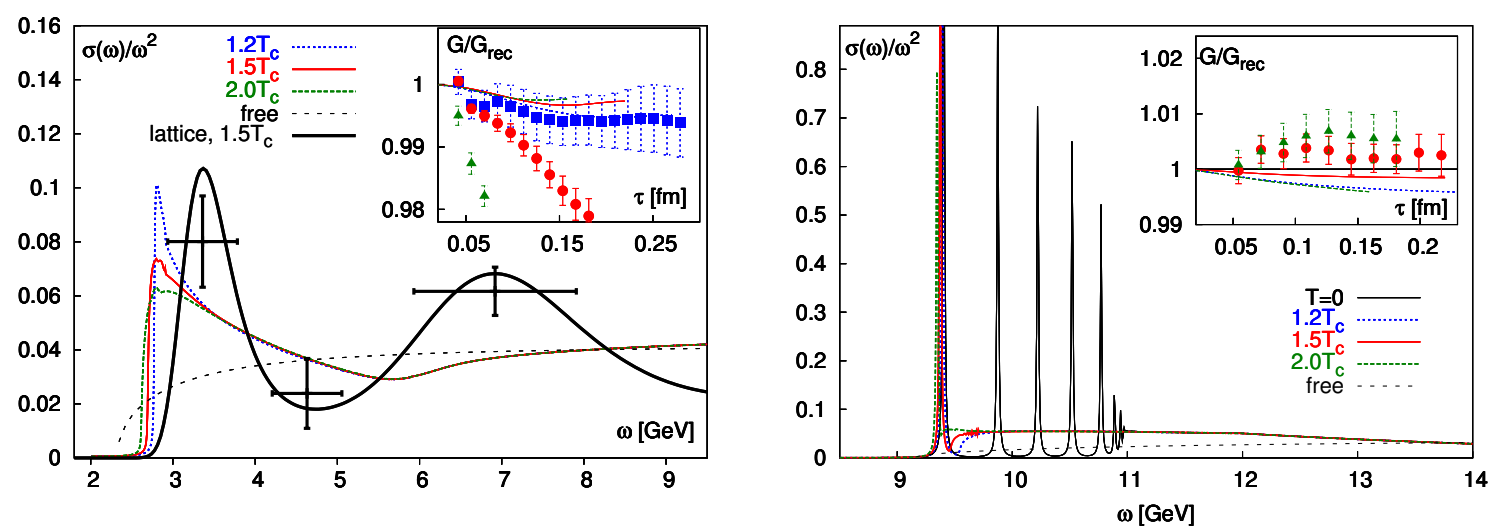

Figure 2: The charmonium (left) and bottomonium (right) spectral functions at different temperatures. For charmonium we also show the spectral functions from lattice QCD obtained from the MEM at $1.5 T_{c}$. The error-bars on the lattice spectral function correspond to the statistical error of the spectral function integrated in the $\omega$-interval corresponding to the horizontal error-bars. The insets show the corresponding ratio $G / G_{\text {rec }}$ together with the results from anisotropic lattice calculations [6]. For charmonium, lattice calculations of $G / G_{r e c}$ are shown for $T=1.2 T_{c}$ (squares), 1.5T (circles), and 2.0T (triangles). For bottomonium lattice data are shown for $T=1.5 T_{c}$ (circles) and $1.8 T_{c}$ (triangles).

of Fig. 2 we also show the corresponding ratio $G / G_{\text {rec }}$. The large changes in the spectral functions are not visible in the correlation functions. These agree quite well with the lattice results. This is because even in the absence of bound states the spectral function is significantly larger than the spectral function corresponding to a freely propagating quark anti-quark pair. In the vector, scalar
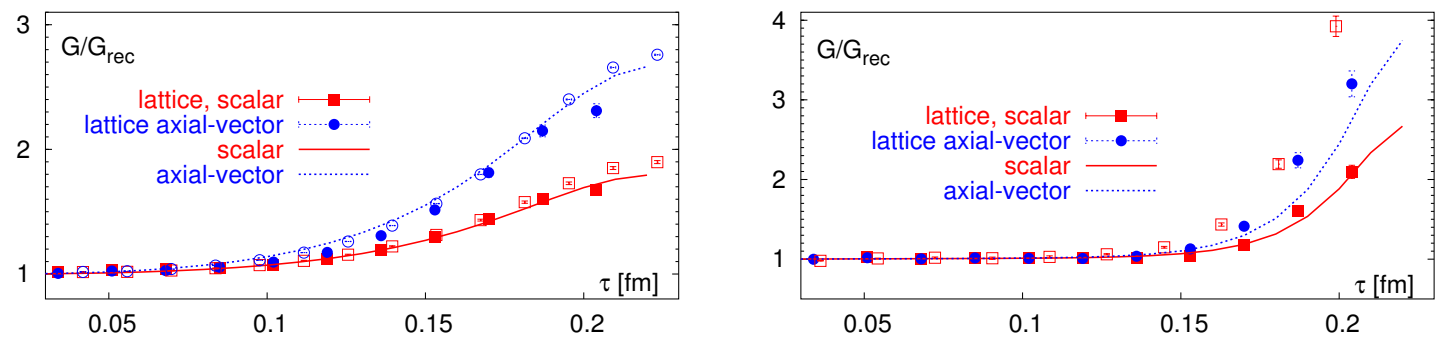

Figure 3: The ratio $G / G_{\text {rec }}$ in the scalar and axial-vector channel at $T=1.5 T_{c}$ for charmonium (left) and bottomonium (right). Lattice calculation on isotropic lattices $[4,29]$ are shown as filled symbols. Open symbols refer to results from anisotropic lattice calculations of Ref. [6].

and axial-vector channels significant temperature dependence has been found [4, 6, 29]. It has been shown that this is due to the zero mode contribution, i.e. due to the $\omega \delta(\omega)$-like contribution to the quarkonium spectral functions [30]. In the vector channel the zero mode contribution corresponds to the heavy quark transport [31]. The zero mode contribution can be estimated in the free case. 
It can also be shown that the zero mode contribution is absent in the pseudo-scalar channel. If we add the free theory result for the zero mode contribution to the spectral function calculated in the potential model we can reproduce the temperature dependence of the scalar and axial-vector correlator both for charmonium and bottomonium. This is demonstrated by Fig. 3 where $G / G_{\text {rec }}$ for the scalar and axial-vector channels is shown.

\section{Conclusions}

We discussed the calculations of quarkonium spectral functions and the corresponding Euclidean time correlators in a potential model. We have found that all quarkonium states, except the $1 S$ bottomonium state, dissolve at temperatures smaller than about $1.2 T_{c}$. This, however, does not lead to significant change of the correlators. Zero mode contribution on the other hand could give a large change in the correlators above the deconfinement transition. We have found that the spectral functions calculated in our model can explain quite well the temperature dependence of the quarkonium correlators obtained in lattice QCD.

\section{Acknowledgments}

This work has been supported by U.S. Department of Energy under Contract No. DE-AC0298CH10886.

\section{References}

[1] T. Matsui and H. Satz, Phys. Lett. B 178 (1986) 416

[2] T. Umeda, K. Nomura and H. Matsufuru, Eur. Phys. J. C 39S1 (2005) 9

[3] M. Asakawa and T. Hatsuda, Phys. Rev. Lett. 92 (2004) 012001

[4] S. Datta, F. Karsch, P. Petreczky and I. Wetzorke, Phys. Rev. D 69 (2004) 094507

[5] G. Aarts, C. R. Allton, R. Morrin, A. P. O. Cais, M. B. Oktay, M. J. Peardon and J. I. Skullerud, arXiv:hep-lat/0610065; Nucl. Phys. A 785 (2007) 198; [arXiv:hep-lat/0608009]; arXiv:0705.2198 [hep-lat]

[6] A. Jakovác, P. Petreczky, K. Petrov and A. Velytsky, Phys. Rev. D 75 (2007) 014506

[7] F. Karsch, et al., Z. Phys. C 37 (1988) 617

[8] G. Röpke, D. Blaschke, H. Schulz, Phys. Rev. D 38 (1988) 3589

[9] T. Hashimoto et al., Z. Phys. C 38 (1988) 251

[10] S. Digal, et al., Phys. Lett. B 514 (2001) 57; Phys. Rev. D 64 (2001) 094015

[11] C. Y. Wong, Phys. Rev. C 72 (2006) 034906

[12] Á. Mócsy and P. Petreczky, Eur. Phys. J. C 43 (2005) 77

[13] Á. Mócsy and P. Petreczky, Phys. Rev. D 73 (2006) 074007

[14] D. Cabrera, R. Rapp, arXiv:hep-ph/0610254

[15] W. M. Alberico, A. Beraudo, A. De Pace and A. Molinari, Phys. Rev. D 75 (2007) 074009 
[16] C. Y. Wong and H. W. Crater, Phys. Rev. D 75 (2007) 034505

[17] O. Kaczmarek, et al., Phys. Lett. B 543 (2002) 41; Phys. Rev. D 70 (2004) 074505

[18] P. Petreczky, K. Petrov, Phys. Rev. D 70 (2004) 054503

[19] O. Kaczmarek, F. Zantow, Phys. Rev. D 71 (2005) 114510

[20] K. Petrov [RBC-Bielefeld Coll.], arXiv:hep-lat/0610041.

[21] O. Kaczmarek, PoS CPOD07 (2007) 043

[22] M. Laine, O. Philipsen, P. Romatschke and M. Tassler, JHEP 0703 (2007) 054

[23] M. Laine, arXiv:0704.1720

[24] F. Karsch, A. Patkós and P. Petreczky, Phys. Lett. B 401 (1997) 69

[25] J. O. Andersen, E. Braaten and M. Strickland, Phys. Rev. Lett. 83 (1999) 2139

[26] J. P. Blaizot, E. Iancu and A. Rebhan, Phys. Rev. Lett. 83 (1999) 2906

[27] Á. Mócsy and P. Petreczky, arXiv:0705.2559 [hep-ph].

[28] Á. Mócsy and P. Petreczky, arXiv:0706.2183 [hep-ph], to be published in Phys. Rev. Lett.

[29] S. Datta, A. Jakovác, F. Karsch and P. Petreczky, AIP Conf. Proc. 842 (2006) 35

[30] T. Umeda, Phys. Rev. D 75 (2007) 094502; PoS (LATTICE 2007) 233

[31] P. Petreczky and D. Teaney, Phys. Rev. D 73 (2006) 014508

[32] W.M. Alberico et. al, arXiv:07062846 